\title{
Stress, Self-Efficacy, Worries, and Challenges for Caregivers of Children with Type 1 Diabetes During the COVID-19 Pandemic
}

Breanne L. Hand ${ }^{1}$, Priya Y. Soni ${ }^{1}$, Rebecca O. Oyetoro ${ }^{2}$, Anastasia Albanese-O'Neill ${ }^{2}$, Heba M. Ismail ${ }^{3}$, Linda A. DiMeglio ${ }^{3}$

${ }^{1}$ Indiana University School of Medicine, ${ }^{2}$ University of Florida College of Medicine, ${ }^{3}$ Indiana University School of Medicine, Division of Pediatric Endocrinology/Diabetology, Department of Pediatrics

\section{Background:}

Approximately 200,000 American youth live with Type 1 Diabetes (T1D). Intensive daily management is critical to optimize disease outcomes. Little is known about COVID-19 pandemic effects on persons with T1D and their caregivers. We hypothesized caregivers would report increased stress, lower self-efficacy (SE), and more difficult diabetes management associated with the pandemic.

Methods: A 49-item survey using Likert scales and open-ended response questions was distributed via email and T1D-related social media platforms from May 4-June 2,2020 to caregivers of children with T1D $<18$ years of age. The Patient-Reported Outcomes Measurement Information System (PROMIS) SE short form was used to measure general SE.

\section{Results:}

260 caregivers completed surveys: $94.2 \%$ female, mean age $42.4 \pm 7.7$ years, $80.3 \%$ college degree or higher, $53.8 \%$ annual income $>\$ 99,000,77.3 \%$ privately insured. Mean child age $11.0 \pm 4.1$ years, mean T1D duration $4.2 \pm 3.4$ years. $24.2 \%$ reported having COVID-19 or knowing someone diagnosed with COVID-19. 71.9\% reported greater stress than before the pandemic. $9.6 \%$ reported job loss, $25.8 \%$ financial difficulty related to COVID-19. General SE levels were high, significantly correlated with overall COVID-19-related SE $(r=0.394, p<0.001)$, and T1D care-SE during COVID-19 ( $r=0.421, p<0.001) .210$ respondents provided open-ended response qualitative information on worries: $87.6 \%$ reported a worry, remainder not worried. Common worries were COVID-19-related diabetes complications including uncontrolled blood sugars, diabetic ketoacidosis, and worry about child's increased risk for severe disease, including death. 209 reported about challenges; 90.9\% stated they had at least one challenge. Family isolation and mental health were the most-stated challenges.

\section{Conclusion:}

Despite reporting high overall SE, caregivers of T1D children during the pandemic reported greater overall stress, worries, and challenges. Given that data do not suggest that children with T1D are at increased risk of COVID-19 complications, healthcare providers should work to address this knowledge gap while providing families with social and mental health supports. 